SUBJECT AREAS:

SEISMOLOGY

GEODYNAMICS

GEOLOGY

VOLCANOLOGY

Received

3 July 2013

Accepted

30 August 2013

Published

17 September 2013

Correspondence and requests for materials should be addressed to

X.L. (lixin@gfzpotsdam.de)

\section{New approach for earthquake/tsunami monitoring using dense GPS networks}

\author{
Xingxing $\mathrm{Li}^{1,2}$, Maorong $\mathrm{Ge}^{1}$, Yong Zhang ${ }^{1,3}$, Rongjiang Wang', Peiliang $\mathrm{Xu}^{4}$, Jens Wickert ${ }^{1}$ \\ \& Harald Schuh'
}

\begin{abstract}
${ }^{1}$ German Research Centre for Geosciences (GFZ), Telegrafenberg, 14473 Potsdam, Germany, ${ }^{2}$ Wuhan University, 129 Luoyu Road, 430079, Wuhan, China, ${ }^{3}$ Institute of Geophysics, China Earthquake Administration, No. 5 MinzudaxueNanlu, Beijing 100081, China, ${ }^{4}$ Disaster Prevention Research Institute, KyotoUniversity, Uji, Kyoto 611 -0011, Japan.
\end{abstract}

In recent times increasing numbers of high-rate GPS stations have been installed around the world and set-up to provide data in real-time. These networks provide a great opportunity to quickly capture surface displacements, which makes them important as potential constituents of earthquake/tsunami monitoring and warning systems. The appropriate GPS real-time data analysis with sufficient accuracy for this purpose is a main focus of the current GPS research. In this paper we propose an augmented point positioning method for GPS based hazard monitoring, which can achieve fast or even instantaneous precise positioning without relying on data of a specific reference station. The proposed method overcomes the limitations of the currently mostly used GPS processing approaches of relative positioning and global precise point positioning. The advantages of the proposed approach are demonstrated by using GPS data, which was recorded during the 2011 Tohoku-Oki earthquake in Japan.

PS (Global Positioning System) has attracted increasing attention and numerous applications in hazard monitoring ${ }^{1-4}$ resulting in a rapid development of high-rate $(\geq 1 \mathrm{~Hz})$ GPS data processing approaches for these applications. High-rate GPS observes displacements directly and thus is particularly valuable in case of large earthquakes/tsunamis ${ }^{5}$. Consequently in the recent years, dense GPS monitoring networks have been built in seismically active regions, e.g., Japan's GEONET (the GPS Earth Observation Network System, http:// www.gsi.go.jp/) and UNAVCO's Plate Boundary Observatory (PBO, http://pbo.unavco.org/). These networks are complementary to seismic monitoring networks and contribute significantly to earthquake/tsunami early warning and hazard risk mitigation ${ }^{3,6,7}$.

Appropriate and precise GPS real-time data analysis is crucial for the use of the network data for hazard monitoring. Currently, the relative baseline/network positioning technique is predominantly used for this purpose $^{3,7-11}$. For moderate-to-short baselines, integer ambiguity resolution can be achieved within a few seconds and sometimes with only one observational epoch to achieve a high positioning accuracy of a few $\mathrm{cm}^{8}$. For the relative positioning (RP) technique, GPS data from a network is analyzed simultaneously to estimate station positions. It is complicated by the need to assign baselines, overlapping Delaunay triangles ${ }^{7}$, or overlapping sub-networks ${ }^{12}$. This is a significant limitation for the challenging simultaneous and precise real-time analysis of GPS data from hundreds or thousands of ground stations. Furthermore, intermittent station dropouts complicate the networkbased relative positioning. Relative positioning also requires a local reference station, which might itself be displaced during a large seismic event, resulting in misleading GPS analysis results. The reference station should be sufficiently far from the focal region, but must also be part of a sub-network that has relatively short baselines. In the case of large earthquakes, such as the Mw 9.0 Tohoku-Oki event at Japan, the reference station may also be significantly displaced, even when it is several hundred kilometers away from the event ${ }^{11}$.

Alternatively, precise point positioning ${ }^{13}$ (PPP) can provide "absolute" displacements with respect to a global reference frame (defined by the satellite orbits and clocks) using a single GPS receiver. It is more flexible than the relative positioning technique and is widely used for hazard monitoring ${ }^{14-17}$. However, the PPP method requires a long convergence period of about 20 minutes after receiver activation or after serious and/or long signal interruption for most of the GPS satellites ${ }^{18}$. The worst case scenario for the GPS component of an earthquake/tsunami monitoring system would be a power failure during the disaster, which would reduce the usefulness of the PPP based displacement solution because of the time required for re-convergence ${ }^{19}$. To avoid this major disadvantage, the PPP regional augmentation ${ }^{20}$ has been developed by making use of atmospheric corrections from a regional reference network to achieve nearly instantaneous ambiguity resolution. But the regional monitoring stations 
could also be displaced by the earthquake. Therefore the current PPP regional augmentation, in which the reference stations are assumed being in static mode and even with known coordinates for generating atmospheric corrections ${ }^{20}$ or pre-fit undifferenced observation residuals ${ }^{25}$, could not be used for earthquake monitoring.

This is our motivation to propose here a novel method for fast or even instantaneous positioning, making full use of the currently available global PPP service and regional GPS monitoring networks. We estimate coordinates of all monitoring stations in kinematic mode to avoid the effects of the earthquake induced-displacements on atmospheric corrections. The derived atmospheric corrections at the stations with fixed ambiguities then can be provided to other monitoring stations for instantaneous ambiguity resolution, so that precise displacements can always be achieved within a few seconds. The series of displacements, derived using the proposed method, will be uninterrupted even in case of a break in tracking (loss of signal lock, cycle slips, or data gaps) due to a power outage or similar disruption. This is a considerable advantage for hazard monitoring application. The new method does not depend on a specific reference station and therefore the analysis results will not be affected by simultaneous shaking of any particular station. It also has better flexibility and efficiency compared to complicated network/subnetwork analysis. We demonstrate the advantages of the novel augmented PPP approach using $1 \mathrm{~Hz}$ GEONET data, collected during the Tohoku-Oki earthquake (Mw 9.0, 11 March, 2011) in Japan.

\section{Results}

The 2011 Mw 9.0 Tohoku-Oki earthquake (11 March 2011, 05:46:23 UTC) in Japan is one of the best GPS recorded large earthquakes, as Japan has one of the densest GPS networks in the world. The Geospatial Information Authority of Japan (GSI) operates more than 1,200 continuously observing GPS stations (collectively called the GPS Earth Observation Network System) all over Japan. The geographical distribution of the stations is indicated in Figure 1. The use of the GEONET data provides an excellent opportunity to evaluate the performance of our novel PPP analysis method. We replayed all the $1 \mathrm{~Hz}$ GPS data collected by the GEONET stations during the 2011 Tohoku-Oki earthquake using the augmented PPP method in simulated real-time mode.

First we process $1 \mathrm{~Hz}$ GPS ground tracking data of about $80 \sim 90$ globally distributed real-time IGS stations using the GFZ's EPOS-RT software in simulated real-time mode for providing GPS orbits, clocks and uncalibrated phase delays (UPD) ${ }^{18,21,23}$ corrections at a $5 \mathrm{~s}$ sampling interval. Using the orbits, clocks and UPD data, the integer ambiguities are fixed in PPP mode for all of the GEONET stations and atmospheric corrections are derived on an individual station basis. For each GEONET station, three nearby GPS stations are selected as augmenting stations. In addition to the orbit, clock and UPD data products from the global PPP service, the atmospheric corrections of the augmenting stations are interpolated and imposed as a constraint on related parameters. Then instantaneous ambiguity

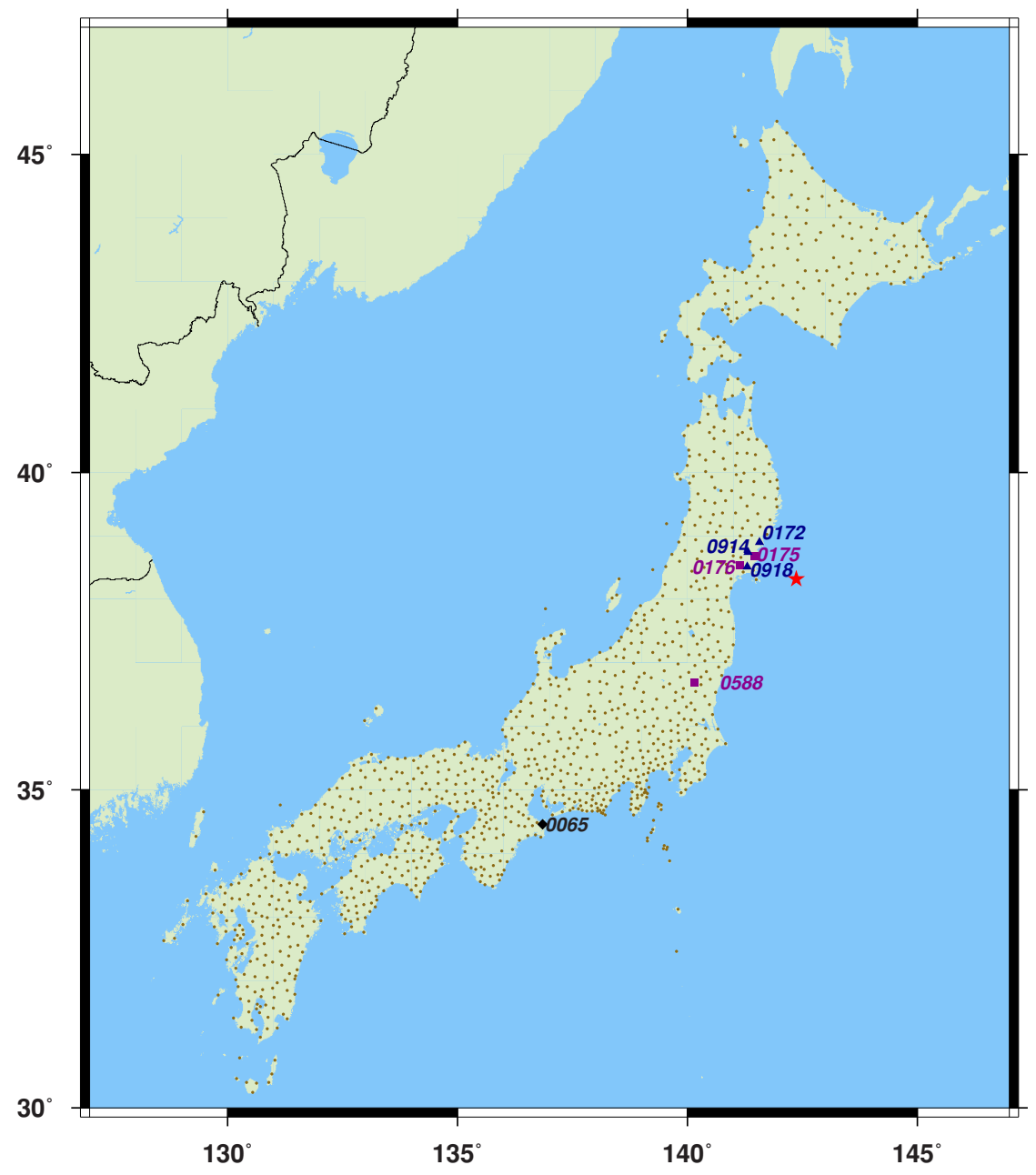

Figure 1 Location of the 2011 Tohoku-Oki earthquake epicenter and the distribution of the high-rate GPS sites. The epicenter is marked by the red star. The brown circles represent GPS sites. The black diamond represents the reference site of relative positioning analysis. The purple rectangles represent the sites of the time series examples. This figure is drawn using GMT software ${ }^{32}$. 
(a) Augmented PPP solution at 0176

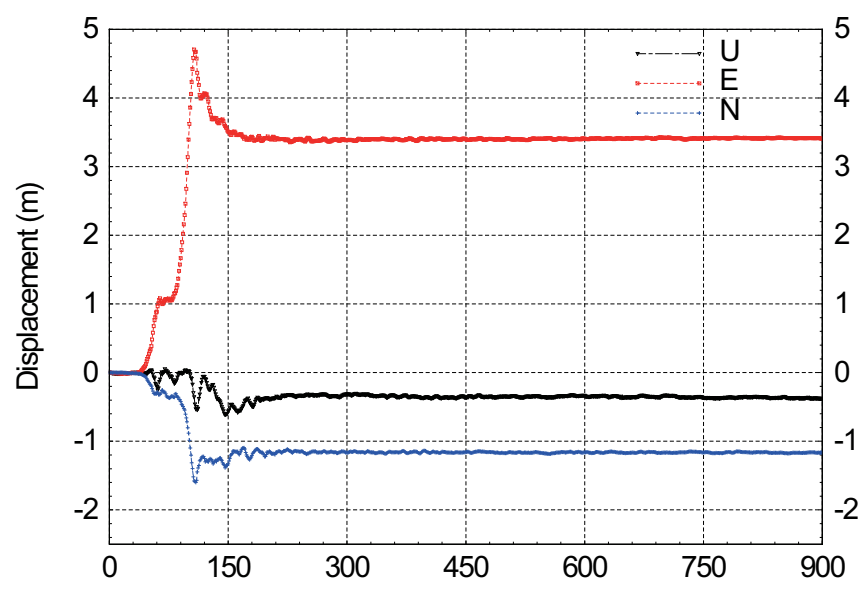

(b) Augmented PPP solution at 0065

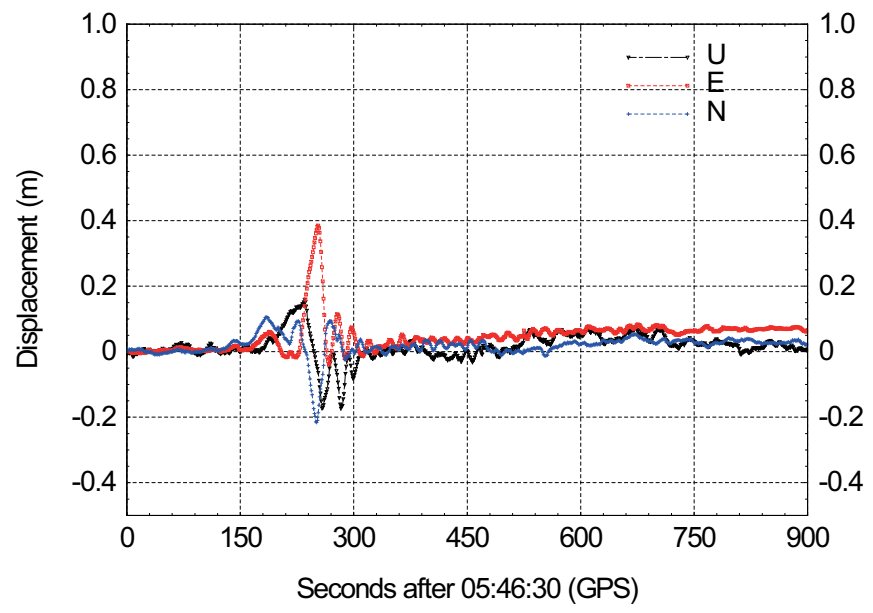

Figure $2 \mid$ The displacement waveforms derived from the augmented PPP solution. The north, east and up components are shown by the blue, red and black curves, respectively. (a) The displacement waveforms at station 0176; (b) The displacement waveforms at the reference station 0065.

fixing is performed independently at each epoch. The displacement waveforms, derived from augmented PPP solution, at station 0176 are shown in the Figure $2 \mathrm{a}$, to illustrate typical behavior. The north, east and up components are respectively shown by the blue, red and black curves.

It is found that there are significant GPS data gaps or cycle slips during the seismic shaking at some GEONET sites (e.g., 0175, 0588, etc). There is for example a data gap of about $2 \mathrm{~min}$ at station 0175 , which starts at epoch 05:47:21 and ends 05:49:27 (GPS time, GPST). The displacements from the augmented PPP solution for station 0175 are shown in Figure 3a. Stations 0172, 0914 and 0918 are selected as the augmenting stations for 0175 . The estimated ionospheric corrections during seismic shaking at the augmenting stations are illustrated in Figure $4 \mathrm{a}$ and Figure 5a. The estimated zenith wet delays during the $600 \mathrm{~s}$ seismic shaking period are respectively $5.4 \pm 0.1 \mathrm{~cm}, 6.5 \pm 0.1 \mathrm{~cm}$ and $6.4 \pm 0.1 \mathrm{~cm}$ at three augmenting stations. With the atmospheric corrections, retrieved from the augmenting stations, the atmospheric delays for 0175 are interpolated using the linear combination method. The resulting interpolations are compared with the estimated values at 0175 in order to assess the accuracy of the interpolation. Figure $4 b$ and Figure $5 b$ show the ionospheric differences between interpolated and estimated values. The differences are found to be smaller than $5 \mathrm{~cm}$. The tropospheric interpolation error is about $0.26 \mathrm{~cm}$. We found that the interpolated (a) Augmented PPP solution at 0175

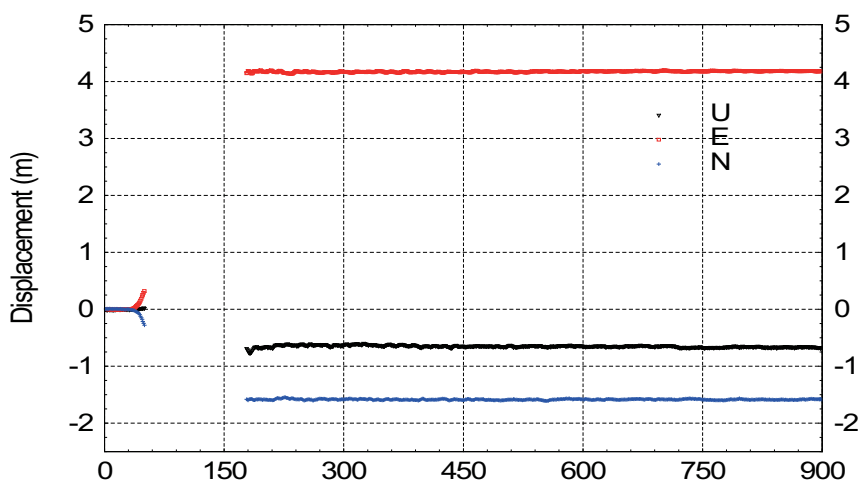

(b) Global PPP solution at 0175

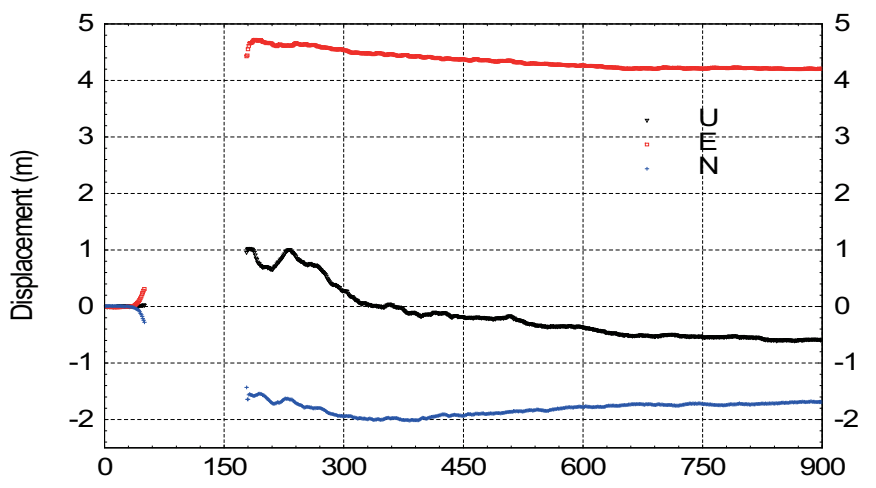

(c) RP solution at 0175

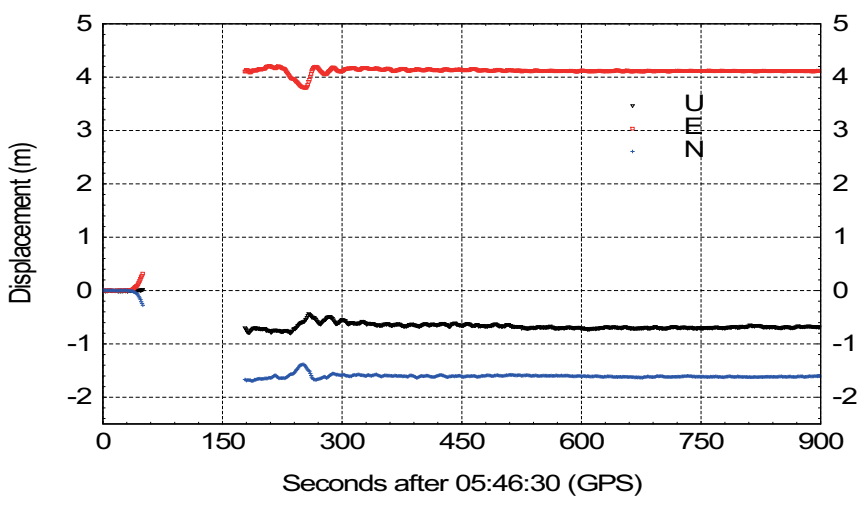

Figure 3 Comparisons of the displacement waveforms derived from augmented PPP, global PPP and relative positioning solutions. The north, east and up components are shown by the blue, red and black curves, respectively. (a) The displacements derived from augmented PPP solution at station 0175 , which has a data gap of about 2 min during the seismic shaking; (b) The displacements derived from the global PPP solution at station 0175 ; (c) The displacements derived from relative positioning at station 0175 , with 0065 as reference station.

atmospheric corrections are accurate enough for rapid ambiguity resolution.

We also derive displacement waveforms of all GEONET stations from the relative positioning (RP) and global PPP solutions, and compare them with the augmented PPP solution. The global PPP displacements for station 0175 are shown in Figure $3 \mathrm{~b}$. In the global PPP solution, the displacement series shows a large disturbance after the data gap that is caused by the convergence sequence for fixing the PPP ambiguities (about $20 \mathrm{~min}$ ). This unstable behavior is an unavoidable problem for a real-time PPP use as the sparse global reference network employed cannot provide accurate atmosphere delays for fast ambiguity resolution. The relative positioning solution 

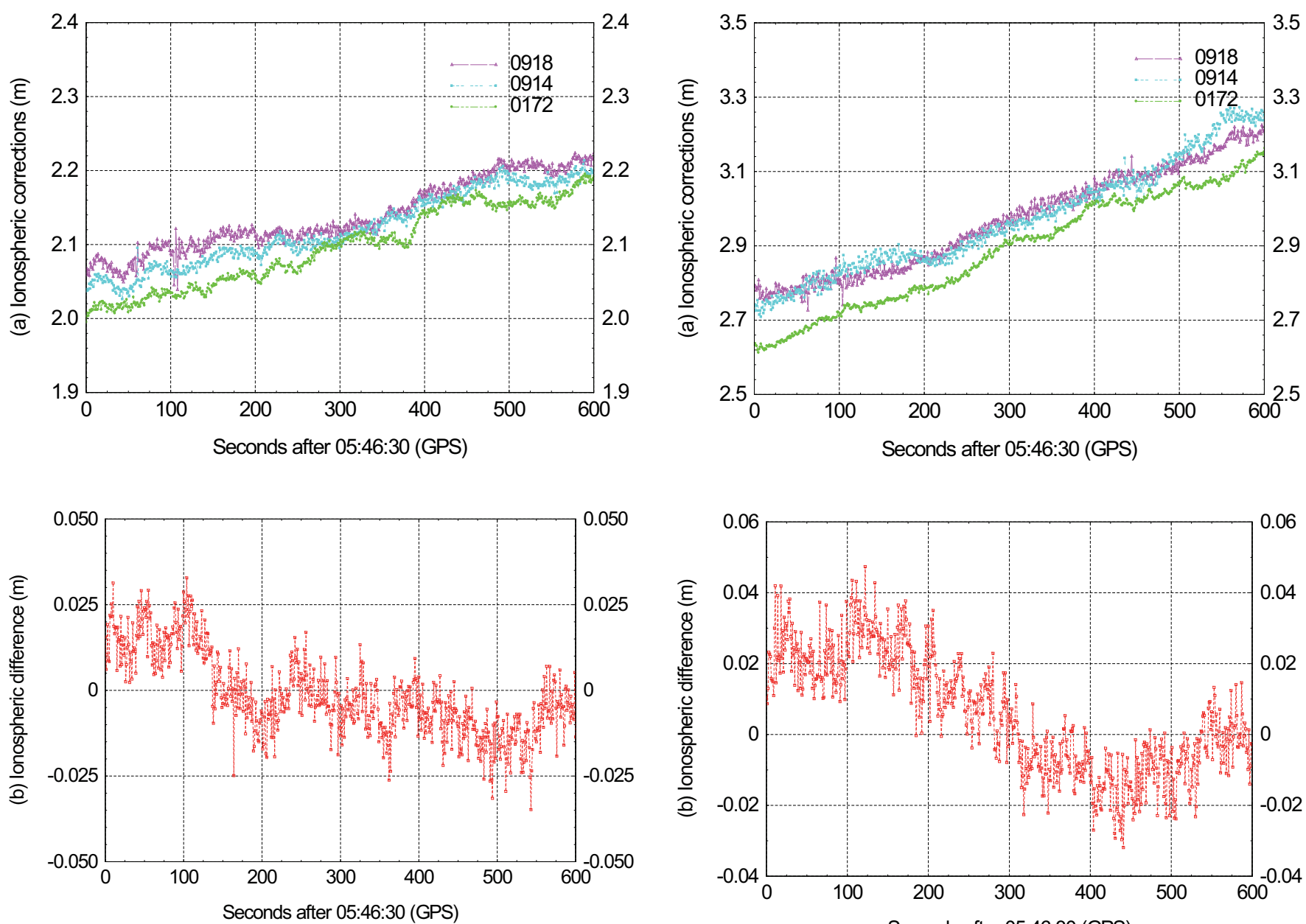

Figure $4 \mid$ Ionospheric corrections at augmenting stations and ionospheric interpolation errors. (a) The estimated ionospheric corrections for GPS satellite PRN 15 at the augmenting stations 0172, 0914 and 0918 during seismic shaking; (b) Ionospheric interpolation errors of PRN 15 for the station 0175.

for the station 0175 is also shown in Figure 3c. For the relative positioning analysis, we adopt the same reference station 0065 as Ohta et al $(2012)^{11}$. It can be seen that there are some fluctuations in the displacement series derived from the relative positioning solution, which are caused by the ground shaking at the reference station location. The Figure $2 \mathrm{~b}$ shows the ground displacements at the 0065 reference station. Peak surface displacements of up to half a meter were recorded at this station during the earthquake even though it is about $700 \mathrm{~km}$ away from the epicenter. The displacement waveforms for the station 0588 derived from augmented PPP (0217, 0590 and 0965 are selected as augmenting stations), global PPP and RP solutions are also compared in Figure 6.

The permanent coseismic displacements of ninety evenly-distributed stations derived from post-processed ARIA solution (5 min solution), real-time augmented PPP, global PPP, and RP solution are shown in Figure 7a, 7b, 7c, and 7d, respectively, by the red arrows. The post-processed ARIA solution is provided by the ARIA team at JPL (Jet Propulsion Laboratory) and Caltech (California Institute of Technology $)^{29}$. It can be found that the permanent coseismic displacements, derived from the real-time augmented PPP solution, are quite consistent with those of post-processed ARIA solution in both horizontal and vertical components. The root mean squared errors (RMS) of the differences between the two solutions are 1.4, 1.1, and $1.7 \mathrm{~cm}$ in north, east, and vertical components, respectively. Figure $7 \mathrm{c}$ shows some significant differences between global PPP

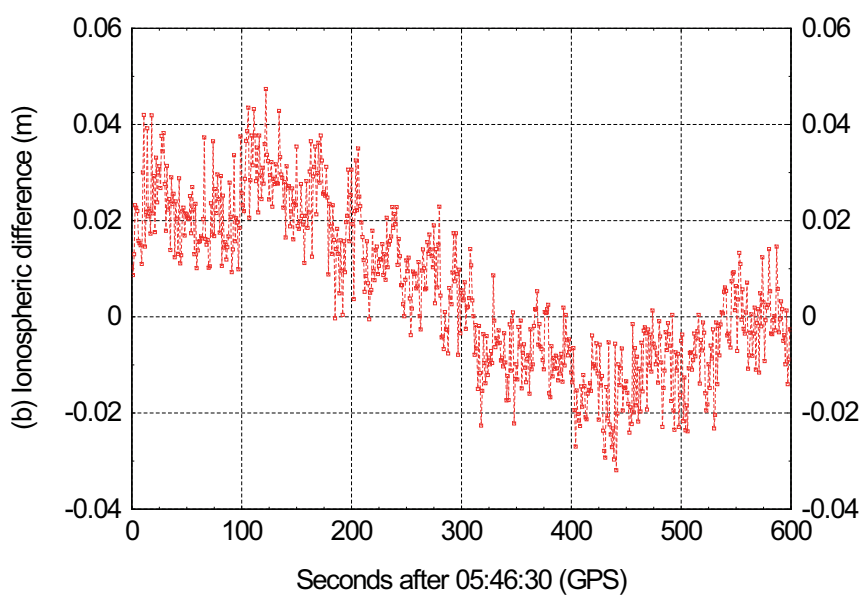

Figure $5 \mid$ Ionospheric corrections for the augmenting stations and the ionospheric interpolation errors. (a) The estimated ionospheric corrections for GPS satellite PRN 28 for the augmenting stations of 0172 , 0914 and 0918 during seismic shaking; (b) Ionospheric interpolation errors of PRN 28 for the station 0175.

and ARIA displacements at some stations, which are caused by the data interruptions at these stations. The corresponding RMS values of the differences are 4.3,22.7, and $9.0 \mathrm{~cm}$ in north, east, and vertical components. Figure 7d shows, that the RP displacements have obvious disagreements with the ARIA results at nearly all stations due to problem of the earthquake shaking of the reference station. The RMS values of the differences are 10.1, 14.1, and $5.7 \mathrm{~cm}$ in north, east, and vertical components. Figure 8 shows the displacement differences between the ARIA solution and the other three solutions. These comparisons show that the augmented PPP method can significantly improve the reliability and accuracy of earthquake-induced coseismic displacements in real-time scenarios.

We derived four fault slip distributions based on the four different GPS analysis techniques introduced above. Identical finite fault parameters are used for the four inversions. Identically as done by Wang et al. $(2013)^{30}$, we employ a slightly curved fault plane, parallel to the assumed subduction slab. The dip angle increases linearly from $10^{\circ}$ on the top (ocean bottom) to $20^{\circ}$ at about $80 \mathrm{~km}$ depth. To avoid any artificial boundary effect, a large enough potential rupture area of $650 \mathrm{~km} \times 300 \mathrm{~km}$ is used. The upper edge of the fault is located along the trench east of Japan, on the boundary between the Pacific plate and the North American plate. The patch size is about $10 \mathrm{~km} \times$ $10 \mathrm{~km}$. The rake angle determining the slip direction at each fault patch is allowed to vary between $90^{\circ} \pm 20^{\circ}$. Green's functions are 
(a) Augmented PPP solution at 0588

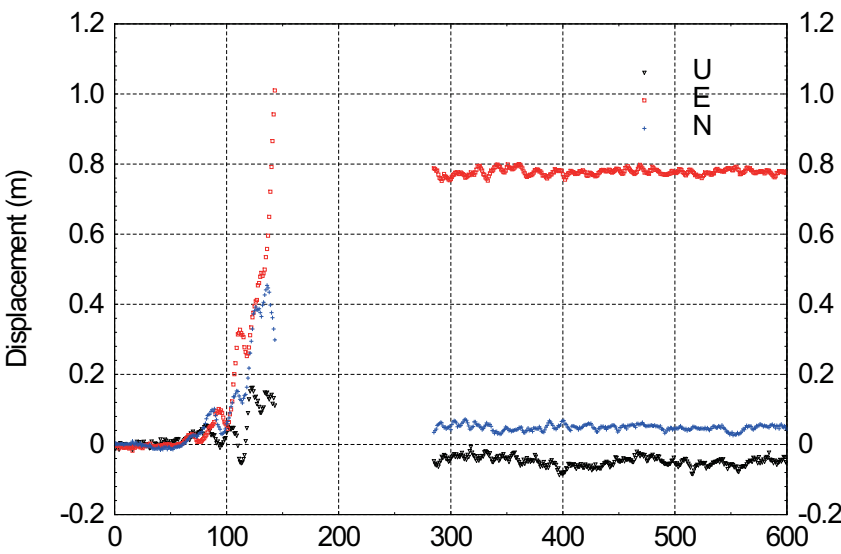

(b) Global PPP solution at 0588

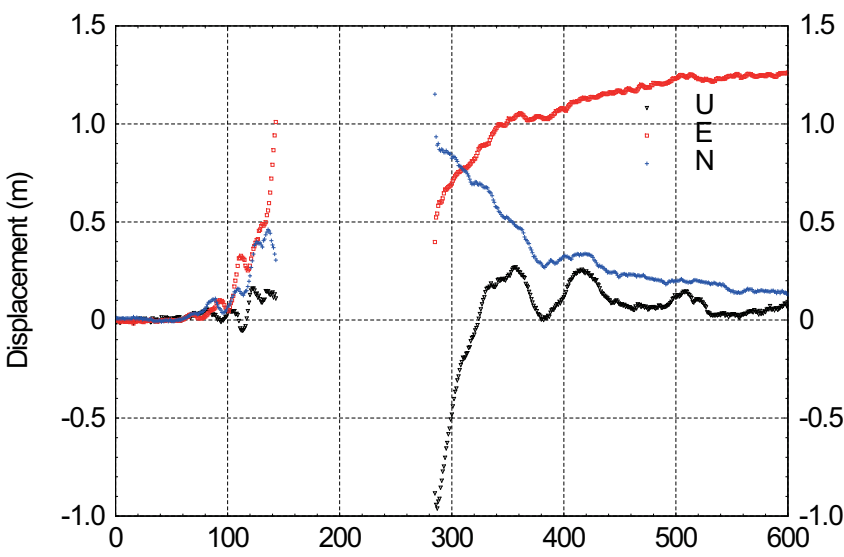

(c) RP solution at 0588

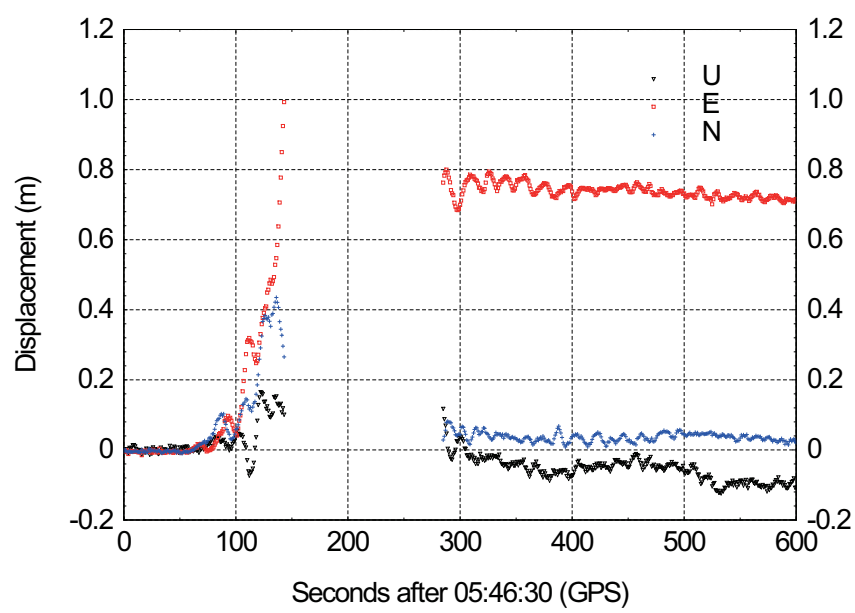

Figure 6 | Comparisons of displacement waveforms derived from augmented PPP, global PPP and RP solutions. The north, east and up components are shown by the blue, red and black curves, respectively. (a) The displacements derived from augmented PPP solution at station 0588, which has a data gap of about 2 min during the seismic shaking; (b) The displacements derived from global PPP solution at station 0588; (c) The displacements derived from relative positioning at station 0588, with 0065 as the reference station.

calculated based on a local CRUST2.0 model by using the software codes from Wang et al. (2003) $)^{31}$.

The comparisons of synthetic and observed displacements on horizontal and vertical components are shown in Figure 7, and the inverted fault slip distributions are shown in Figure 9. Although the four results show similar slip distribution, the inversion from real-time augmented PPP solution is the most consistent with post-processed ARIA solution not only for the slip distribution, but also for the displacement fittings. Supposing that the post-processed ARIA result is the most reliable and can be taken as a reference for other three results, the inversion of global PPP has the worst slip distribution, and the inversion of RP solution has the worst displacement fittings. Figure 10 shows the fault slip differences between the ARIA solution and the other three solutions. Overall, the comparison of the inversions shows that the augmented PPP method is beneficial for fault slip inversion in real-time scenarios. It provides a more accurate and robust estimation of the fault slip distribution and displacement fittings than the global PPP solution and RP solution. By contrast, the global PPP and RP solutions result in relatively poor slip distributions not only in peak slip, but also in the extension of the slip areas (Figure 10).

\section{Discussion}

We proposed a new GPS analysis method for hazard (e.g. earthquake and tsunami) monitoring. The new augmented PPP method can overcome the limitations of current relative positioning and global PPP approaches for this application. The performance of the new approach is evaluated by GPS ground network data, observed during the 2011 Tohoku-Oki earthquake in Japan.

The atmospheric corrections retrieved from the nearby monitoring stations can be interpolated with accuracy better than $5 \mathrm{~cm}$. This means that the interpolated atmospheric corrections are accurate enough for rapid ambiguity resolution, which is a prerequisite to achieve the most precise displacements. The displacement waveforms, derived using the augmented PPP approach are immune to the convergence problem caused by data gaps and cycle slips and the problem of the earthquake shaking the reference station compared to the waveforms based on RP and global PPP analysis. This makes augmented PPP potentially appropriate for the application in operational earthquake/tsunami monitoring and warning systems. The reliability and accuracy of permanent coseismic displacements are also significantly improved. The RMS accuracy of about 1.4, 1.1, and $1.7 \mathrm{~cm}$ are achieved in the north, east, and vertical components, respectively. The inversion results indicate that the augmented PPP solution is the most consistent with post-processed ARIA solution both in the fault slip distribution and displacement fittings.

\section{Methods}

Successful resolution of integer-cycle carrier-phase ambiguities is a prerequisite to achieve the most precise position estimates with GPS by transforming precise but ambiguous phase range measurements into precise unambiguous measurements ${ }^{21,22}$ For relative positioning, the uncalibrated phase delays (UPD) are removed by the application of the double-difference (DD) technique and thus the phase ambiguity can be fixed to integers ${ }^{21}$. The atmospheric delays are also mostly eliminated in case of moderate-to-short baselines, so that integer-cycle phase ambiguities can be fixed within few seconds. Recent studies show that the UPDs can be estimated with high accuracy and reliability from a global reference network and transferred to the GPS monitoring station to allow resolution of the ambiguities without differencing ${ }^{18,23}$. Several international GNSS service (IGS) analysis centers provide GPS orbit, clock, and UPD data products to allow real-time PPP use enabling ambiguity resolution anywhere in the world ${ }^{24-26}$. However, PPP still needs a comparatively long (re)convergence time of approximate 20 minutes to achieve reliable integer ambiguity resolution because precise atmospheric delay models cannot be derived from such a sparse global reference network ${ }^{18}$

An increasing number of regional GPS monitoring networks are installed around the world for precise navigation and geophysical applications, especially in seismically active regions (e.g. Japan, Western North America, Greece, and Chile). One possible solution for achieving fast ambiguity resolution in PPP is to retrieve the atmospheric delays as corrections from data of these dense regional networks. By applying the UPD corrections, the integer un-differenced ambiguities on the L1 and L2 frequencies can be fixed in PPP mode at all regional monitoring stations. The atmospheric corrections of the ionospheric slant and tropospheric zenith wet delay then can be derived from the PPP fixed solution as

$$
I_{r, j}^{s}-m_{r}^{s} \cdot Z_{r}+u_{r}^{s} \cdot \Delta r=-l_{r, j}^{s}-t^{s}+t_{r}+\lambda_{j}\left(b_{r, j}-b_{j}{ }^{s}\right)+\lambda_{j} N_{r, j}^{s}+\varepsilon_{r, j}^{s}
$$


(a)

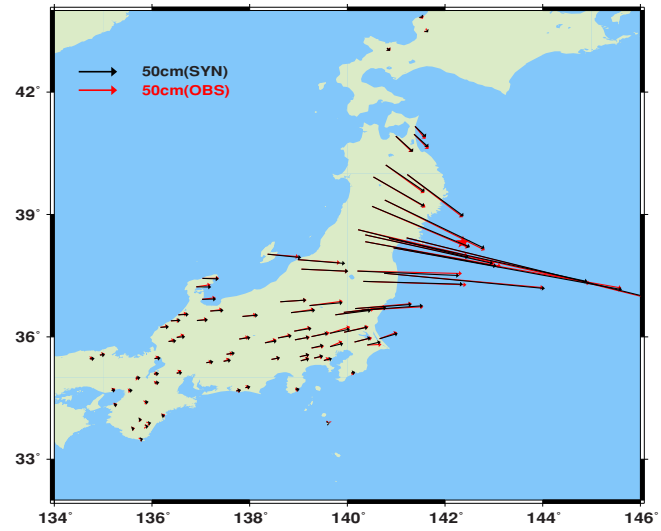

(b)

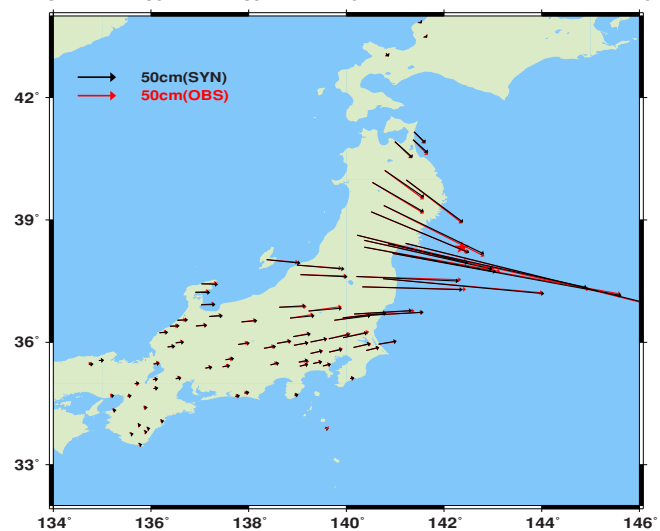

(c)

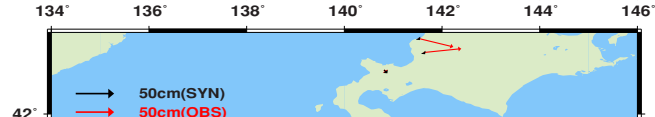

(d)
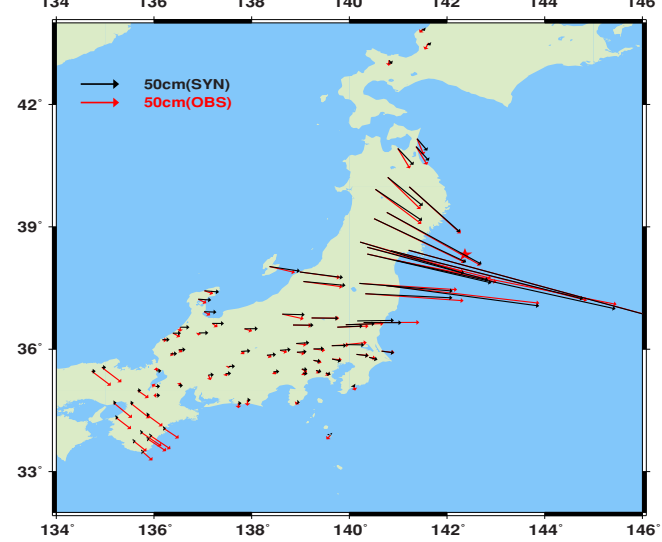
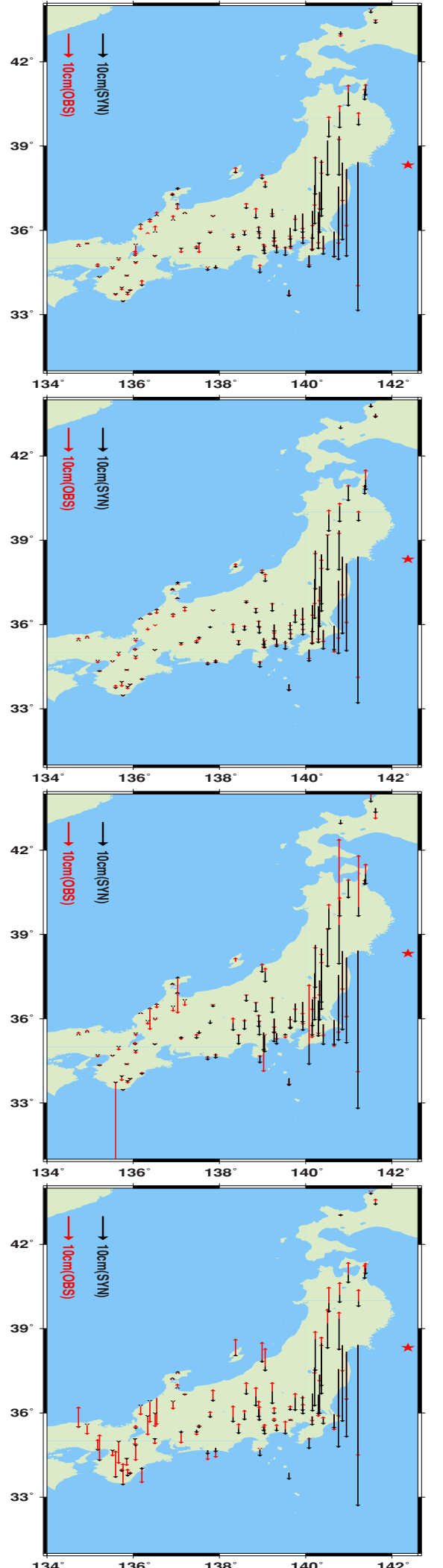

Figure 7 The comparisons of observed and synthetic displacements in horizontal components, and in vertical components, respectively. (a) Inversion with the post-processed ARIA solution; (b) Inversion with coseismic displacements obtained from real-time augmented PPP solution; (c) Inversion with real-time global PPP solution; (d) Inversion with real-time RP solution. This figure is drawn using GMT software ${ }^{32}$. 
(a)
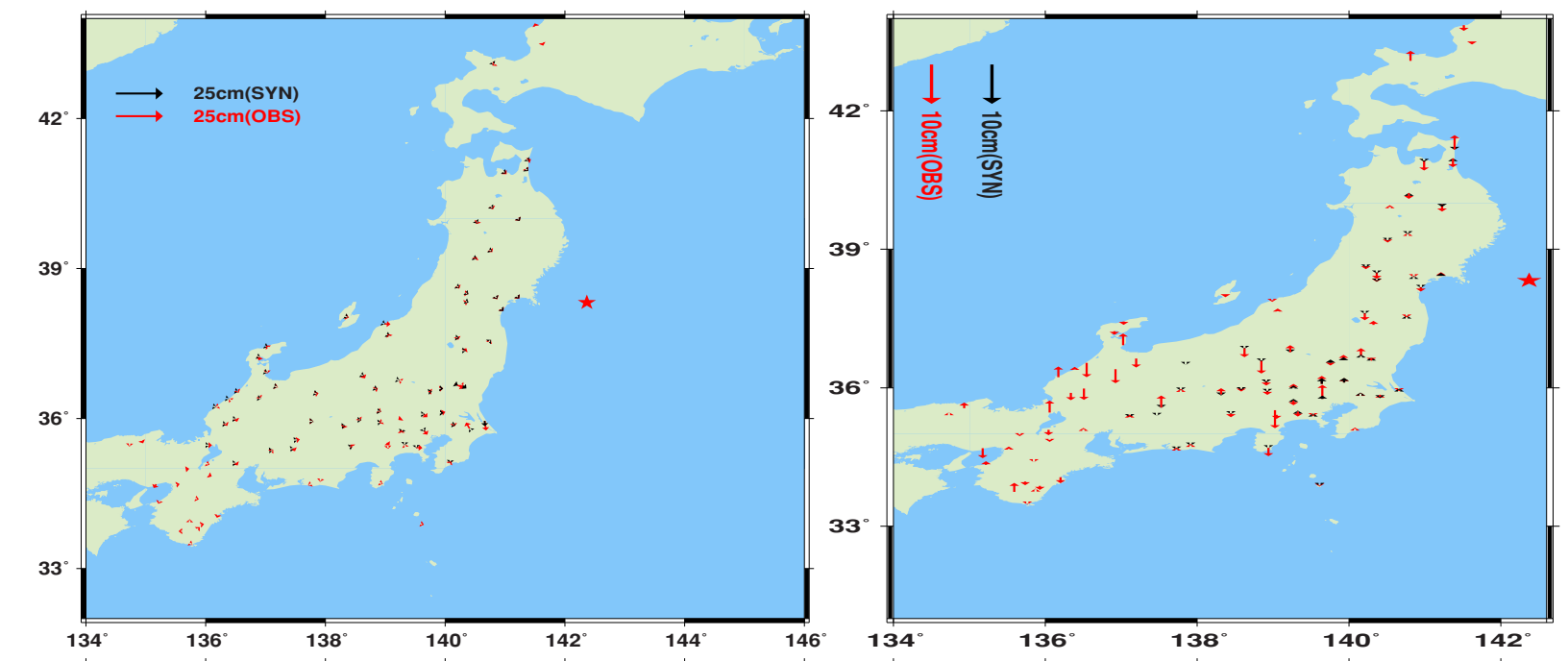

(b)
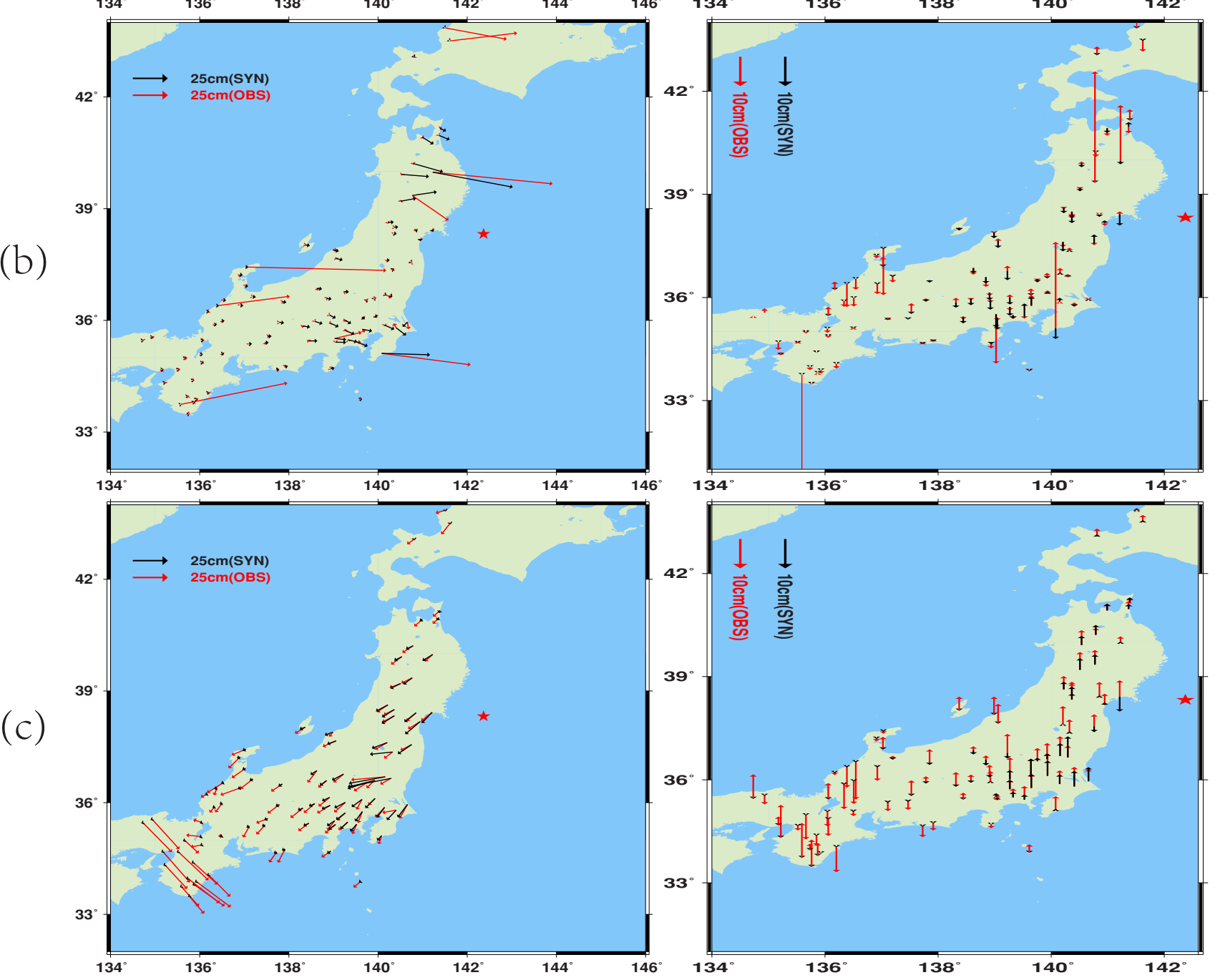

Figure $8 \mid$ The residual displacements from the ARIA solution. (a) Residual differences between augmented PPP and ARIA vectors; (b) Residual differences between global PPP and ARIA vectors; (c) Residual differences between RP and ARIA vectors. This figure is drawn using GMT software ${ }^{32}$.

$$
-I_{r, j}^{s}-m_{r}^{s} \cdot Z_{r}+u_{r}^{s} \cdot \Delta r=-p_{r, j}^{s}-t^{s}+t_{r}+c\left(d_{r, j}+d_{j}^{s}\right)+e_{r, j}^{s}
$$

Where, $l_{r, j}^{s}, p_{r, j}^{s}$ denote phase and code observables from satellite $s$ to receiver $r$ at frequency $j$; $u_{r}^{s}$ is the unit direction vector from site to satellite; $\Delta r$ denotes the increments of the receiver positions; $Z_{r}$ denotes the tropospheric zenith wet delay; $m_{r}^{s}$ is the wet mapping function; $t^{s}$ and $t_{r}$ are the clock errors; $\lambda_{j}$ is the wavelength; $b_{r j}$ is the receiver-dependent uncalibrated phase delay; $b_{j}^{s}$ is the satellite-dependent UPD; $d_{r, j}$ and $d_{j}^{s}$ are the code biases; $I_{r, j}^{s}$ is the ionospheric delay; $N_{r, j}^{s}$ is the integer phase ambiguity; $e_{r, j}^{s}$ and $\varepsilon_{r, j}^{s}$ are the measurement noise terms of the pseudo-range and carrier phase.

This procedure is very flexible and computational efficient to be applied even for monitoring networks with a large number of stations as the atmospheric corrections are derived for each station individually. Because regional monitoring stations themselves could be displaced by the earthquake, the coordinates are estimated in kinematic mode to avoid the effects of earthquake induced-displacements on the atmospheric corrections that are generated. The constraints imposed on the 
(a)
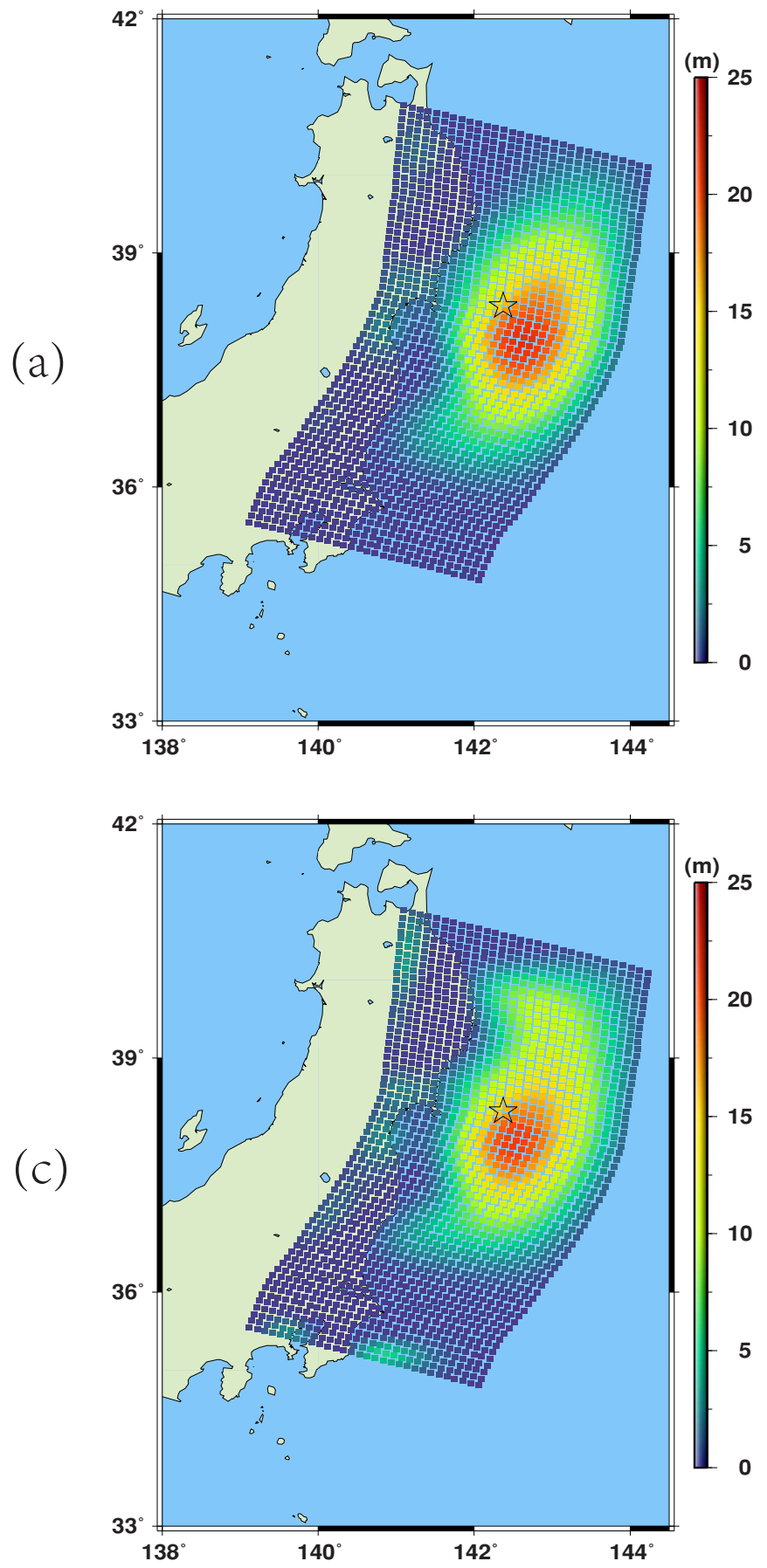

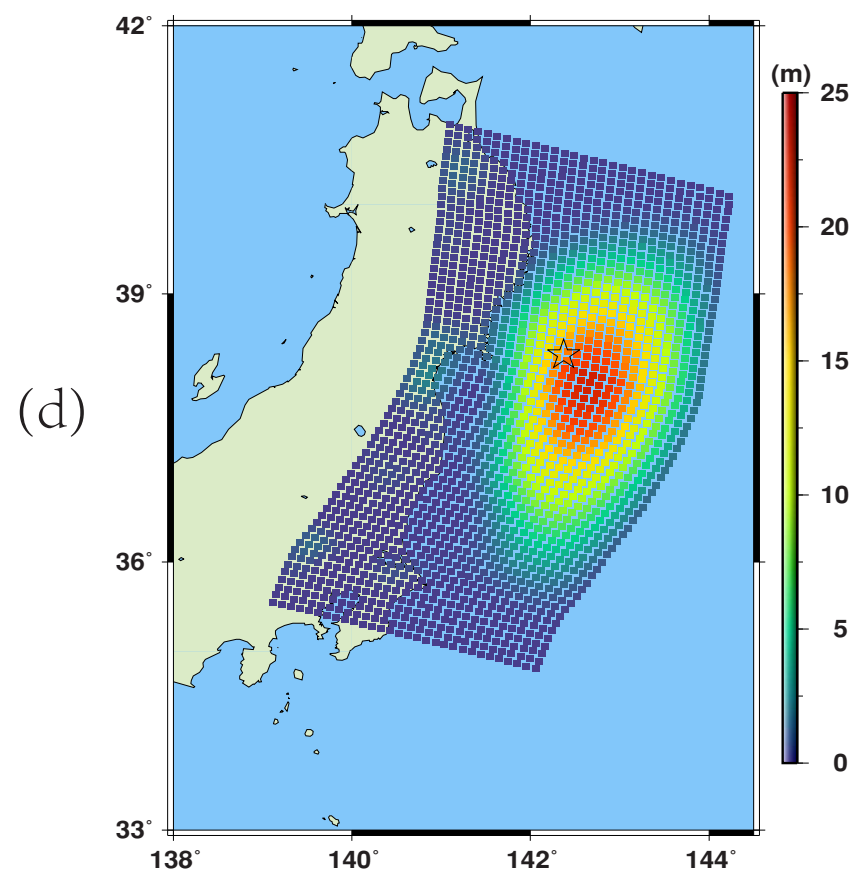

(b)

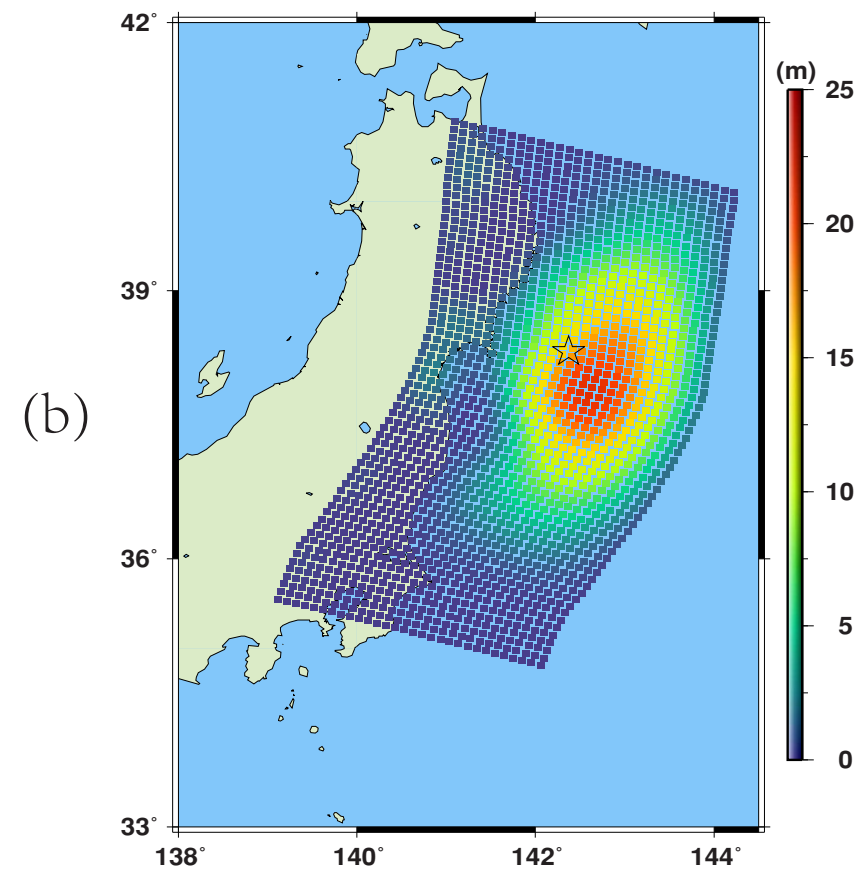

(1)

15

(1)

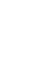

Figure 9 | The inverted fault slip distributions. (a) Inversion with post-processed ARIA solution; (b) Inversion with coseismic displacements obtained from real-time augmented PPP solution; (c) Inversion with real-time global PPP solution; (d) Inversion with real-time RP solution. The star denotes the epicenter. This figure is drawn using GMT software ${ }^{32}$.

kinematic coordinates of adjacent epochs are fine-tuned by using an adaptive filter ${ }^{27}$ in real-time to strengthen the solution. Usually atmospheric delay is rather stable over short periods and can be represented by a constant or a linear function. Therefore, even in periods of strong shaking, station position and atmosphere are distinguishable in parameter estimation because of the significant difference in their temporal characters.

A polynomial model can be used to represent the derived atmospheric corrections on small regional scales. Here three or more nearby monitoring stations are selected as augmenting stations for each monitoring station, and the atmospheric corrections of the selected augmenting stations are interpolated by a Linear Combination Method $(\mathrm{LCM})^{28}$ as

$$
\sum_{i=1}^{n} \alpha_{i}=1, \sum_{i=1}^{n} \alpha_{i}\left(\hat{X}_{m}-\hat{X}_{i}\right)=0, \sum_{i=1}^{n} \alpha_{i}^{2}=\operatorname{Min}
$$


(a)

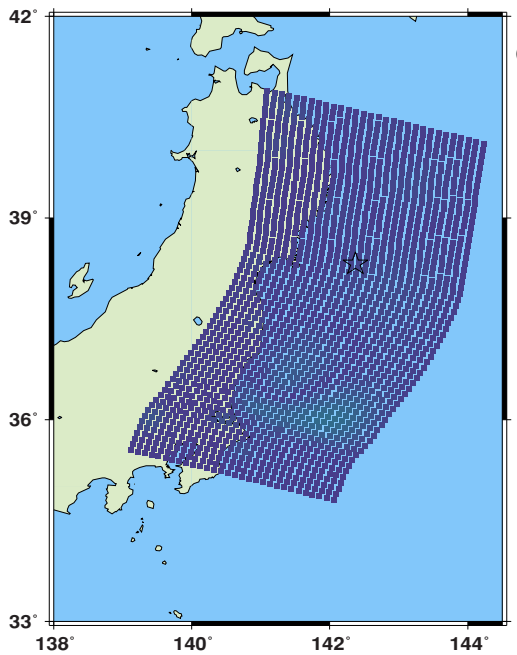

(b)

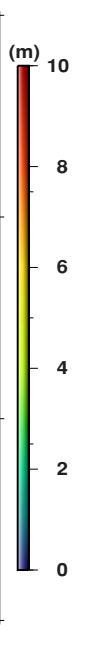

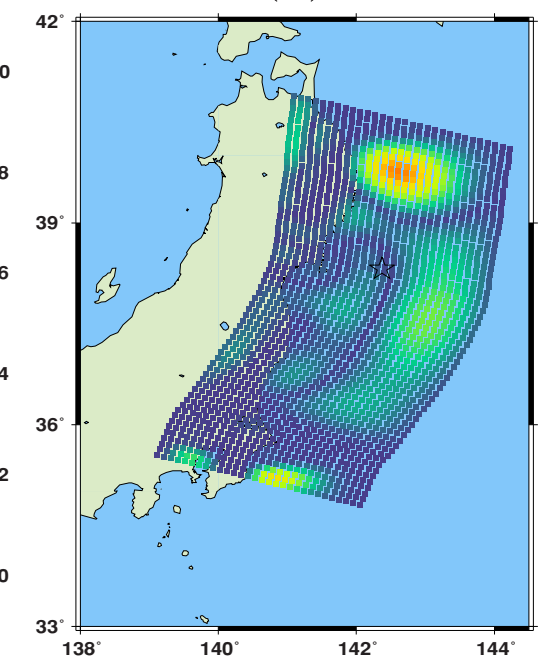

(c)

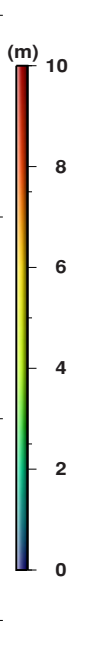

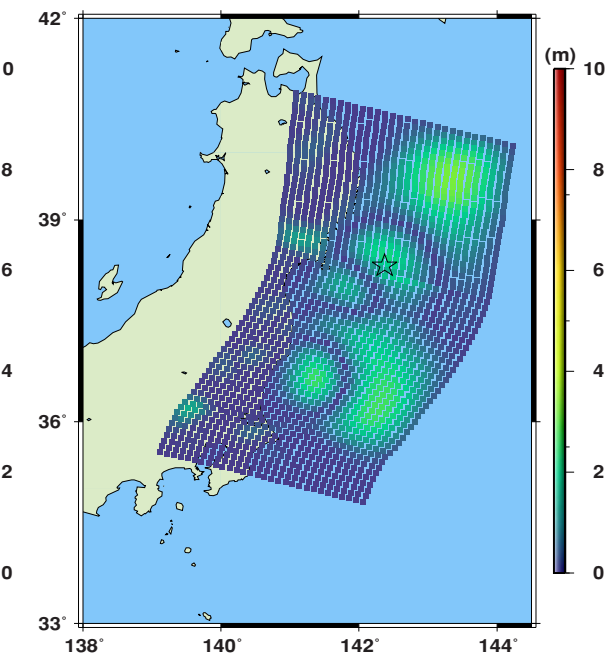

Figure $10 \mid$ The residual slip distributions from the ARIA solution. (a) Residual differences between augmented PPP and ARIA inversion; (b) Residual differences between global PPP and ARIA inversion; (c) Residual differences between RP and ARIA inversion. The star denotes the epicenter. This figure is drawn using GMT software ${ }^{32}$.

Assuming that $r_{1}$ to $r_{n}$ are selected as augmenting stations for interpolating corrections for the monitoring station $r_{m}$. The ionospheric slant delay parameter for an individual satellite $s_{i}$ is constrained to the interpolated correction as

$$
I_{r_{m}}^{s_{i}}-\tilde{I}_{r_{1}, r_{2} \cdots r_{n}}^{s_{i}}=w_{I}, \quad w_{I} \sim N\left(0, \sigma_{w_{I}}^{2}\right)
$$

And the constraint for the zenith wet delay parameter is

$$
Z_{r_{m}}-\tilde{Z}_{r_{1}, r_{2} \cdots r_{n}}=w_{T}, \quad w_{T} \sim N\left(0, \sigma_{w_{T}}^{2}\right)
$$

Where $I_{r_{m}}^{s_{i}}$ denotes the slant ionospheric delay from station $r_{m}$ to satellite $s_{i} ; \tilde{I}_{r_{1}}^{s_{i}, r_{2} \ldots r_{n}}$ is the interpolated ionospheric correction; $Z_{r_{m}}$ denotes the zenith wet delay for station $r_{m}$, and $\tilde{Z}_{r_{1}, r_{2} \cdots r_{n}}$ is the interpolated correction. $w_{I}$ and $w_{T}$ are the biases between the true and the interpolated atmospheric corrections. The statistical processes of $w_{I}$ and $w_{T}$ are zero mean white processes with variance of $\sigma_{w_{I}}^{2}$ and $\sigma_{w_{T}}^{2}$ for the ionospheric and tropospheric delays, respectively.

By adding this precise atmospheric delay model to the orbit, clock and UPD products used in global PPP ambiguity resolution, instantaneous ambiguity resolution is achievable at the monitoring station, so that the augmented PPP can have ambiguity resolution performance equivalent to relative positioning. It should be mentioned that the selection of augmenting stations is critical, as atmospheric corrections can only be derived from augmenting stations at which the ambiguity resolution is successfully achieved.

1. Hirahara, K., Nakano, T. \& Hoso, Y. An experiment for GPS strain seismometer. in Proc. of the Japanese Symposium on GPS, 15-16 December, Tokyo, Japan, 67-75 (1994).

2. Ge, L. et al. GPS Seismometers with up to $20 \mathrm{~Hz}$ Sampling Rate. Earth Planets Space. 52, 881-884 (2000).

3. Blewitt, G., Kreemer, C., Hammond, W. C. \& Plag, H. P. Rapid determination of earthquake magnitude using GPS for tsunami warning systems. Geophys. Res. Lett. 33, L11309 (2006).

4. Larson, K. GPS seismology. J. Geodesy 83, 227-233 (2009).

5. Allen, R. \& Ziv, A. Application of real-time GPS to earthquake early warning. Geophys. Res. Lett. 38, L16310 (2011).

6. Blewitt, G. et al. GPS for real-time earthquake source determination and tsunami warning systems. J. Geodesy 83, 335-343 (2009).

7. Crowell, B., Bock, Y. \& Squibb, M. Demonstration of earthquake early warning using total displacement waveforms from real time GPS networks. Seismol. Res. Lett. 80, 772-782 (2009).

8. Bock, Y., Nikolaidis, R., de Jonge, P. J. \& Bevis, M. Instantaneous geodetic positioning at medium distances with the global positioning system. J. Geophys. Res. 105, 233-253 (2000)

9. Larson, K., Bodin, P. \& Gomberg, J. Using 1-Hz GPS data to measure deformations caused by the Denali fault earthquake. Science 300, 421-424 (2003).

10. Melgar, D., Bock, Y. \& Crowell, B. W. Real-Time Centroid Moment Tensor Determination for Large Earthquakes from Local and Regional Displacement Records. Geophys. J. Int. 188, 703-718 (2012).
11. Ohta, Y. et al. Quasi real-time fault model estimation for near-field tsunami forecasting based on RTK-GPS analysis: Application to the 2011 Tohoku-Oki earthquake (Mw 9.0). J. Geophys. Res. 117, B02311 (2012).

12. Bock, Y., Melgar, D. \& Crowell, B. W. Real-Time Strong-Motion Broadband Displacements from Collocated GPS and Accelerometers. Bull. Seism. Soc. Am. 101, 2904-2925 (2011).

13. Zumberge, J. F., Heflin, M. B., Jefferson, D. C., Watkins, M. M. \& Webb, F. H. Precise point positioning for the efficient and robust analysis of GPS data from large networks. J. Geophys. Res. 102, 5005-5017 (1997).

14. Kouba, J. Measuring seismic waves induced by large earthquakes with GPS. Stud. Geophys. Geod. 47, 741-755 (2003).

15. Wright, T. J., Houlié, N., Hildyard, M. \& Iwabuchi, T. Real-time, reliable magnitudes for large earthquakes from $1 \mathrm{~Hz}$ GPS precise point positioning: The 2011 Tohoku-Oki (Japan) earthquake. Geophys. Res. Lett. 39, L12302 (2012).

16. Li, X. et al. Real-time high-rate co-seismic displacement from ambiguity-fixed precise point positioning: Application to earthquake early warning. Geophys. Res. Lett. 40, 295-300 (2013).

17. $\mathrm{Xu}$, P. et al. High-rate precise point positioning (PPP) to measure seismic wave motions: an experimental comparison of GPS PPP with inertial measurement units. J. Geodesy 87, 361-372 (2013).

18. Li, X., Ge, M., Zhang, H. \& Wickert, J. A method for improving uncalibrated phase delay estimation and ambiguity-fixing in real-time precise point positioning. J. Geodesy 87, 405-416 (2013).

19. Collins, P. et al. Precise Point Positioning for Real-Time Determination of CoSeismic Crustal Motion. Proc. of ION GNSS-2009, Savannah, Georgia, 22-25 September, 2479-2488 (2009).

20. Li, X., Zhang, X. \& Ge, M. Regional reference network augmented precise point positioning for instantaneous ambiguity resolution. J. Geodesy 85, 151-158 (2011).

21. Blewitt, G. Carrier phase ambiguity resolution for the Global Positioning System applied to geodetic baselines up to $2000 \mathrm{~km}$. J. Geophys. Res. 94, 10187-10203 (1989).

22. Dong, D. N. \& Bock, Y. Global Positioning System network analysis with phase ambiguity resolution applied to crustal deformation studies in California. J. Geophys. Res. 94, 3949-3966 (1989).

23. Ge, M., Gendt, G., Rothacher, M., Shi, C. \& Liu, J. Resolution of GPS carrier-phase ambiguities in precise point positioning (PPP) with daily observations. J. Geodesy 82, 389-399 (2008).

24. Dow, J. M., Neilan, R. E. \& Rizos, C. The International GNSS Service in a changing landscape of Global Navigation Satellite Systems. J. Geodesy 83, 191-198 (2009).

25. Ge, M., Dousa, J., Li, X., Ramatschi, M. \& Wickert, J. A Novel Real-Time Precise Positioning Service System: Global Precise Point Positioning with Regional Augmentation. JGPS 11, 2-10 (2012).

26. Loyer, S., Perosanz, F., Mercier, F., Capdevilleet, H. \& Marty, J. Zero-difference GPS ambiguity resolution at CNES-CLS IGS Analysis Center. J. Geodesy 86, 991-1003 (2012).

27. Yang, Y., He, H. \& Xu, G. Adaptively robust filtering for kinematic geodetic positioning. J. Geodesy 75, 109-116 (2001).

28. Han, S. Carrier phase-based long-range GPS kinematic positioning. $\mathrm{PhD}$ thesis, School of Geomatic Engineering, The University of New South Wales (1997). 
29. Simons, M. et al. The 2011 magnitude 9.0 Tohoku-Oki earthquake: Mosaicking the megathrust from seconds to centuries. Science 322, 1421-1425 (2011).

30. Wang, R. et al. The 2011 Mw 9.0 Tohoku-Oki Earthquake: Comparison of GPS and Strong-Motion Data. Bull. Seism. Soc. Am. 103, 1336-1347 (2013).

31. Wang, R., Lorenzo-Martín, F. \& Roth, F. Computation of deformation induced by earthquakes in a multi-layered elastic crust - FORTRAN programs EDGRN/ EDCMP. Comput Geosci 29, 195-207 (2003).

32. Wessel, P. \& Smith, W. H. F. New, improved version of generic mapping tools released. Eos Trans. AGU 79, 579-579 (1998).

\section{Acknowledgments}

Thanks go to the International GNSS Service (IGS) for providing GPS data of globally distributed reference stations. $1 \mathrm{~Hz}$ GEONET data was provided by the Geospatial

Information Authority of Japan.

\section{Author contributions}

X.L. and M.G. initial idea and conception; X.L., M.G., Y.Z. and R.W. wrote the main manuscript text; P.X., J.W. and H.S. helped with the writing. All authors reviewed the manuscript.

\section{Additional information}

Competing financial interests: The authors declare no competing financial interests. How to cite this article: Li, X. et al. New approach for earthquake/tsunami monitoring using dense GPS networks. Sci. Rep. 3, 2682; DOI:10.1038/srep02682 (2013).

(c) (1) (2) (2) This work is licensed under a Creative Commons AttributionNonCommercial-ShareAlike 3.0 Unported license. To view a copy of this license, visit http://creativecommons.org/licenses/by-nc-sa/3.0 